\title{
Model parameter identification from measurement data as a prerequisite for dynamic torque calibration - Measurement results and validation
}

\author{
Leonard Klaus \\ Physikalisch-Technische Bundesanstalt, Bundesallee 100, 38116 Braunschweig, Germany
}

\begin{abstract}
The dynamic calibration of torque transducers requires the modelling of the measuring device and of the transducer under test. The transducer's dynamic properties are described by means of model parameters, which are going to be identified from measurement data. To be able to do so, two transfer functions are calculated. In this paper, the transfer functions and the procedure for the model parameter identification are presented. Results of a parameter identification of a torque transducer are also given, and the validity of the identified parameters is analysed by comparing the results with independent measurements. The successful parameter identification is a prerequisite for a model-based dynamic calibration of torque transducers.
\end{abstract}

Section: RESEARCH PAPER

Keywords: mechanical modelling; model-based calibration; linear and time invariant system

Citation: Leonard Klaus, Model parameter identification from measurement data as a prerequisite for dynamic torque calibration - Measurement results and validation, Acta IMEKO, vol. 5, no. 3, article 9, November 2016, identifier: IMEKO-ACTA-05 (2016)-03-09

Editor: Paolo Carbone, University of Perugia, Italy

Received February 5, 2016; In final form August 5, 2016; Published November 2016

Copyright: (C) 2016 IMEKO. This is an open-access article distributed under the terms of the Creative Commons Attribution 3.0 License, which permits unrestricted use, distribution, and reproduction in any medium, provided the original author and source are credited

Funding: This work was part of the Joint Research Project IND09 Traceable Dynamic Measurement of Mechanical Quantities of the European Metrology Research Programme (EMRP). The EMRP is jointly funded by the EMRP participating countries within EURAMET and the European Union

Corresponding author: Leonard Klaus, e-mail: leonard.klaus@ptb.de

\section{INTRODUCTION}

Several applications with dynamic torque excitation require traceable measurement. At present, only standards and procedures for the static calibration of torque transducers exist. Static calibration is an insufficient base for an analysis of dynamic measurements in terms of measurement uncertainties and influences from dynamic signal components. To be able to describe the dynamic influences of a torque transducer on a measurement set-up and vice versa, a corresponding calibration is mandatory [1]. Therefore, a measuring device and procedures for a dynamic characterisation of torque transducers were developed in the context of a joint European research project [2].

This paper is a substantially extended version of a contribution to the XXI IMEKO World Congress 2015 [2] and includes additional measurement results and analyses for the validation of the correctness of the identified parameters.
The focus of this contribution is to demonstrate that a model parameter identification technique can be applied to identify parameters of a torque transducer from measurement data. For this purpose, parameter estimation results are validated by means of independent measurements and additional measurements with a modified set-up.

A measurement uncertainty evaluation for the identified parameters will be covered in a separate publication, as it would be significantly beyond the scope of this article.

\section{DYNAMIC TORQUE MEASURING DEVICE}

The measurement principle of the dynamic torque measuring device (depicted in Figure 1) is based on Newton's second law. The product of a known static mass moment of inertia $J$ of a body and a measured time-dependent angular acceleration $\ddot{\varphi}(t)$ equals the time-dependent torque: 


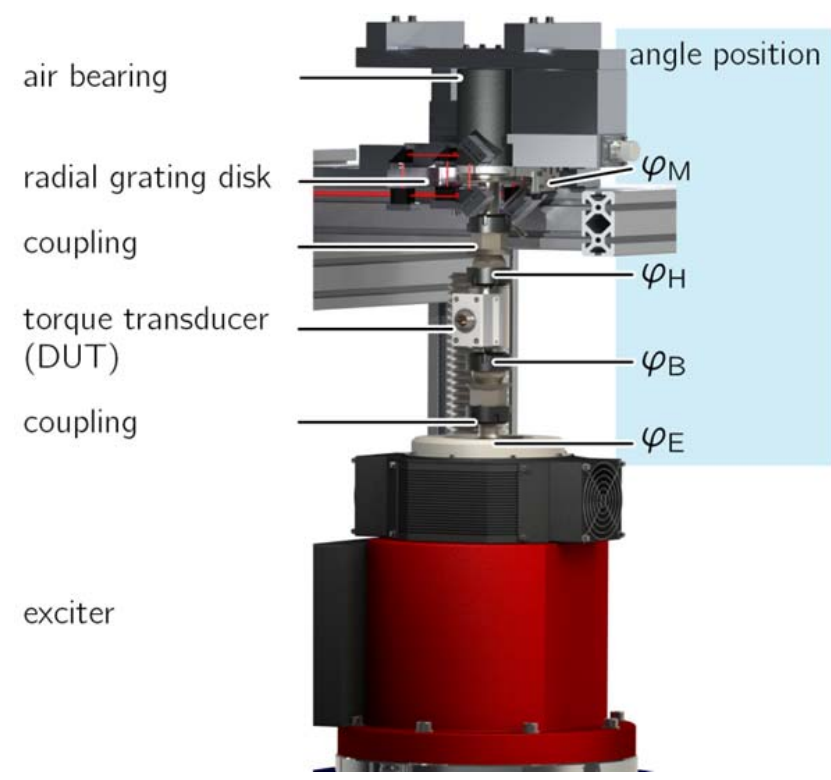

Figure 1. Dynamic torque measuring device with the different components arranged vertically on top of the exciter.

$M(t)=J \cdot \ddot{\varphi}(t)$.

The measuring device consists of a rotatable vertical shaft assembly, on which all essential components are arranged in series.

At the bottom, a rotational exciter generates a forced excitation by means of sinusoidal oscillations. The torque transducer under test (device under test, DUT) is arranged on top of the exciter between two coupling elements. These couplings are designed to be both torsionally stiff and compliant for parasitic bending moments and axial loads simultaneously. At the top, the arrangement of the DUT and the couplings is followed by an angular grating disk for the angular acceleration measurement and an air bearing to prevent axial loads acting on the components of the drive shaft.

The angle position at the top $\left(\varphi_{\mathrm{M}}\right)$ is measured by means of a laser Doppler vibrometer and the radial grating disk. The angular acceleration at the bottom $\left(\ddot{\varphi}_{\mathrm{E}}\right)$ is measured by an angular accelerometer embedded in the rotor of the rotational exciter.

\section{MODELLING}

The dynamic behaviour of the transducer under test is described by a linear time-invariant (LTI) model. It is based on the mechanical design of typical strain gauge torque transducers. The model consists of two rigid mass moment of inertia elements (MMOI) which are connected by a massless torsional spring and damper in parallel. A sole modelling of the transducer under test is not sufficient, because of the fact that the dynamic behaviour of torque transducers can be influenced by the coupled components. Torque transducers are always coupled to their mechanical environment at both sides which causes a bidirectional influence on the dynamic behaviour of the torque transducer and the coupled mechanical components (which are always arranged in some type of drive assembly).

To be able to identify the model parameters of the DUT, the modelling was expanded from the model of the transducer to a model of the whole measuring device, which is the mechanical environment in the case of the calibration. The model again consists of mass moment of inertia elements, torsional springs and dampers, assuming the LTI behaviour of the measuring device. It is based on the mechanical design of the components of the drive shaft. The representation of the model components and the corresponding components of the measuring device are given in Figure 2.

This model can be described by a set of inhomogeneous ordinary differential equations (ODEs). With the mass moment of inertia matrix $\boldsymbol{J}$, the torsional stiffness matrix $\boldsymbol{K}$ and the damping matrix $\boldsymbol{D}$ there follows

\section{$\boldsymbol{J} \cdot \ddot{\boldsymbol{\varphi}}+\boldsymbol{D} \cdot \dot{\boldsymbol{\varphi}}+\boldsymbol{K} \cdot \boldsymbol{\varphi}=\boldsymbol{M}$.}

The angle vector $\boldsymbol{\varphi}$ describes the angle excitations at different positions in the model; its derivatives $\dot{\boldsymbol{\varphi}}$ and $\ddot{\boldsymbol{\varphi}}$ represent the angular velocity and angular acceleration, respectively. The load vector $\boldsymbol{M}$ describes the excitation of the rotational exciter.

Based on this equation system, the model parameter identification will be carried out.

The excitation signals chosen are monofrequent sinusoids. With these harmonic waveforms, not all necessary angle position, angular velocity and angular acceleration data has to be derived independently or by numerical differentiation / integration but can be calculated as follows:

$\varphi(t)=\hat{\varphi} \cdot \mathrm{e}^{i \omega t}$,

$\dot{\varphi}(t)=i \omega \hat{\varphi} \cdot \mathrm{e}^{i \omega t}=i \omega \varphi(t)$,

$\ddot{\varphi}(t)=-\omega^{2} \hat{\varphi} \cdot \mathrm{e}^{i \omega t}=-\omega^{2} \varphi(t)$,

where $i=\sqrt{-1}$ denotes the imaginary number.

Measurements are not possible at all angle positions given in (2) and depicted in Figure 2. Technically, measurements at the positions $\varphi_{\mathrm{H}}$ and $\varphi_{\mathrm{B}}$ cannot be carried out, because sufficiently precise measurement components would require elaborating adjustments after each mounting and dismounting of the transducer under test. To gather the information necessary for parameter identification, the output signal of the transducer $U_{\text {DUT }}$ is used as an indicator for the difference in the torsion angle above and below the transducer. This assumption is valid,

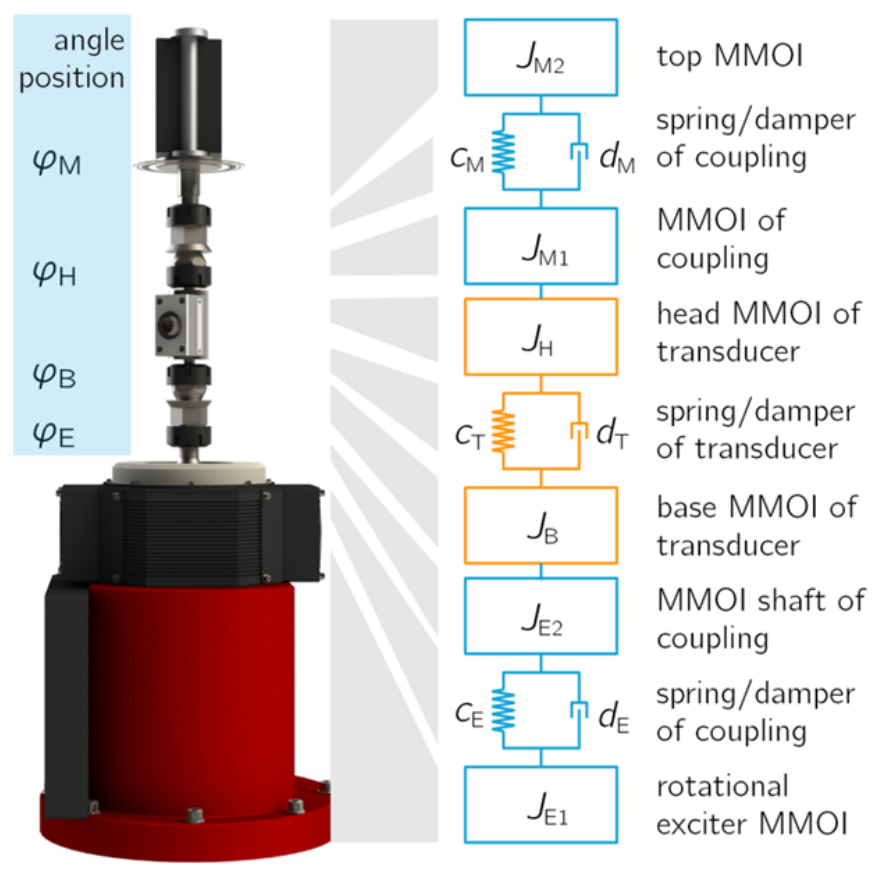

Figure 2. Dynamic torque measuring device (left) and corresponding mode representation (right) of the measuring device (blue) and the DUT (orange). 
because it is assumed that the output signal of the transducer is proportional to its torsion $\Delta \varphi_{\mathrm{HB}}=\varphi_{\mathrm{H}}-\varphi_{\mathrm{B}}$ giving

$U_{\mathrm{DUT}}(t) \propto \Delta \varphi_{\mathrm{HB}}(t)$.

During calibration, three measurement signals are acquired simultaneously: The angle position $\varphi_{\mathrm{M}}(t)$ at the top, the angular acceleration $\ddot{\varphi}_{\mathrm{E}}(t)$ at the bottom, and the voltage output $U_{\text {DUT }}(t)$ of the transducer under test. These signals will be processed to determine the magnitude and phase of each harmonic and monofrequent signal by means of a sine fit.

\section{KNOWN AND UNKNOWN MODEL PARAMETERS}

A prerequisite for the model parameter identification of the unknown parameters of the transducer under test is a sufficiently low number of unknown model parameters of the system. To this end, the necessary parameters of the measuring device were determined in advance. The properties of the measuring device will not change for different transducers, and therefore needed to be determined only once. Three auxiliary measurement set-ups for the measurement of the mass moment of inertia, torsional stiffness and rotational damping were developed [3]-[5] and the corresponding properties of the measuring device's components were determined. The only model parameters to remain unknown prior to the model parameter identification are the parameters of the transducer under test.

\section{MODEL PARAMETER ESTIMATION}

The model parameter identification is carried out based on transfer functions. Generally, a transfer function $H(\mathrm{i} \omega)$ describes the relation between input $X(\mathrm{i} \omega)$ and output $Y(\mathrm{i} \omega)$ of a system as follows

$H(\mathrm{i} \omega)=\frac{Y(\mathrm{i} \omega)}{X(\mathrm{i} \omega)}$.

A model parameter estimation based on such a transfer function is carried out by approximating a best set of parameters of the model function to the measured data. This set of parameters is characterised by giving the lowest deviations of model assumptions and measurement data.

However, due to measurement uncertainty deviations, which will always occur, an exact measurement of the different input quantities $X(\mathrm{i} \omega), Y(\mathrm{i} \omega)$ will not be possible. The measured data will always be disturbed randomly due to measurement errors. The measurement data $X_{\mathrm{M}}(\mathrm{i} \omega), Y_{\mathrm{M}}(\mathrm{i} \omega)$ is therefore modelled as multivariate random numbers with estimated state of knowledge probability density functions (PDF). Figure 3 depicts the relation of the measured input and output quantities and a model transfer function for the general example from (5).

Applying this general approach to the dynamic torque

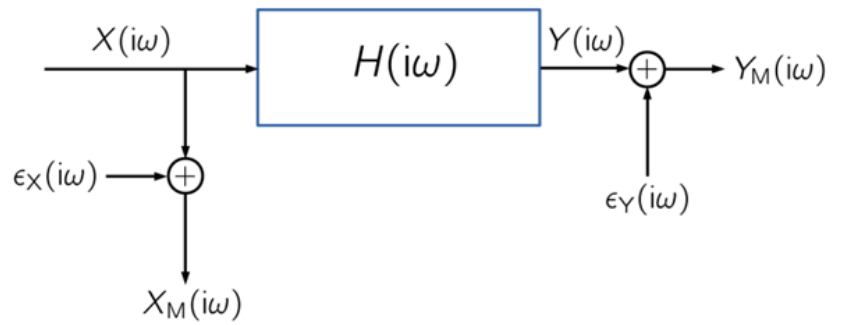

Figure 3. Schematic illustration of a transfer function and its relation to input and output. calibration, two complex transfer functions of the system are calculated based on the three available signals acquired during calibration measurements. One transfer function $H_{\text {top }}(\mathrm{i} \omega)$ describes the dynamics of the top part of the measuring device giving

$H_{\text {top }}(\mathrm{i} \omega)=\frac{\rho \cdot \Delta \varphi_{\mathrm{HB}}(\mathrm{i} \omega)}{\ddot{\varphi}_{\mathrm{M}}(\mathrm{i} \omega)}$

with the (still unknown) proportionality factor $\rho$ linking the voltage output of the transducer to its torsion (cf. equation (4)).

The same applies to the bottom part of the measuring device giving the transfer function:

$H_{\text {bott }}(\mathrm{i} \omega)=\frac{\rho \cdot \Delta \varphi_{\mathrm{HB}}(\mathrm{i} \omega)}{\ddot{\varphi}_{\mathrm{E}}(\mathrm{i} \omega)}$.

The two transfer functions are illustrated in Figure 4. The underlying ODE system gives the corresponding equations for both transfer functions. Each transfer function contains the unknown parameters of the transducer under test. The parameters $\rho, J_{\mathrm{H}}, d_{\mathrm{T}}, c_{\mathrm{T}}$, can be derived from $H_{\mathrm{top}}$, while $H_{\mathrm{bott}}$ contains all the wanted parameters: $\rho, J_{\mathrm{H}}, J_{\mathrm{B}}, d_{\mathrm{T}}$, and $c_{\mathrm{T}}$. The derivation of the model parameters from the ODE system and the relations with the two transfer functions is thoroughly described in [6]. The model parameter identification is carried out based on both of the two transfer functions.

The more information is taken into account for the model parameter estimation, the more reliable the outcome can be. Based on the chosen estimator, knowledge about the input quantities, the output quantities, and about the parameters to be identified prior to the estimation (so-called a priori knowledge) can be considered.

Three typical estimators are compared regarding their demand of knowledge of the different quantities in Table 1. A ' + ' indicates necessary information about the distribution of a quantity; a ' - ' indicates that it is not necessary to know the distribution.

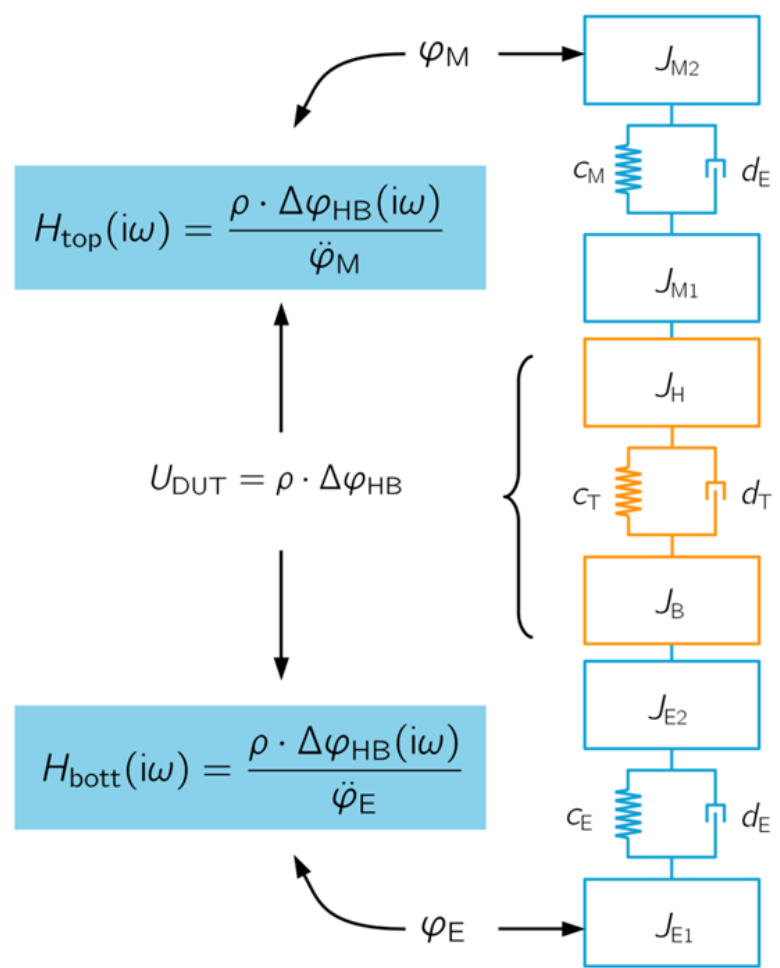

Figure 4. The two calculated transfer functions based on the acquired measurands. 
Table 1. Requirements of different estimators.

\begin{tabular}{l|cc}
$\begin{array}{l}\text { knowledge about } \rightarrow \\
\text { estimator } \downarrow\end{array}$ & $\begin{array}{l}\text { distribution of } \\
\text { input quantities }\end{array}$ & $\begin{array}{l}\text { distribution of the } \\
\text { (unknown) } \\
\text { parameters }\end{array}$ \\
\hline $\begin{array}{l}\text { Bayes' estimator } \\
\begin{array}{l}\text { maximum likelihood } \\
\text { estimator } \\
\text { least squares estimator }\end{array}\end{array}$ & + & + \\
& - & -
\end{tabular}

The classical frequentist approach to a model parameter estimation takes no uncertainty contributions in terms of PDFs of input quantities into account. Therefore, the estimated set of parameters consists of the estimated values, but not of information about their distribution. The least squares (LS) estimator and the maximum likelihood estimator are frequentist estimation approaches.

The least squares estimator minimises the squared sum of residuals of the measured transfer function data $H_{\text {top, }}$, $H_{\text {bott,M }}$ and of a model function $\boldsymbol{G}$. The data of the two transfer functions is merged in a data vector $\boldsymbol{Y}_{\mathrm{D}, i}\left(\mathrm{i} \omega_{i}\right)=\left[H_{\mathrm{top}, \mathrm{M}, i}\left(\mathrm{i} \omega_{i}\right), H_{\mathrm{bott}, \mathrm{M}, i}\left(\mathrm{i} \omega_{i}\right)\right]^{T}$ for each $i$ of $n$ data points, the same applies to the model function $\boldsymbol{G}$ which consists of the model representations of the two mentioned transfer functions $H_{\text {top }}\left(\mathrm{i} \omega, \boldsymbol{\theta}_{1}, \boldsymbol{\theta}_{2}\right)$ and $H_{\text {bott }}\left(\mathrm{i} \omega, \boldsymbol{\theta}_{1}, \boldsymbol{\theta}_{2}\right)$ accordingly, giving the least squares estimator:

$\widehat{\boldsymbol{\theta}}_{2}=\arg \min _{\widehat{\boldsymbol{\theta}}_{2}} \sum_{i=1}^{n}\left(\boldsymbol{Y}_{\mathrm{D}, i}\left(\mathrm{i} \omega_{i}\right)-\boldsymbol{G}\left(\mathrm{i} \omega_{i}, \boldsymbol{\theta}_{1}, \boldsymbol{\theta}_{2}\right)\right)^{2}$.

The estimation result is denoted with a hat $(\hat{\bullet})$. In the case of the model parameter estimation of torque transducers, the model function consists of a vector of parameters of the measuring device $\boldsymbol{\theta}_{1}$, which is known, and the vector of unknown parameters of the transducer under test $\boldsymbol{\theta}_{2}$, which is estimated. No weighting of the different values is applied with this estimator.

The likelihood function $\ell$ for a vector of samples $\boldsymbol{x}=$ $\left[x_{1}, x_{2}, \ldots, x_{n}\right]^{T}$ with the sample's PDF $p\left(x_{i}, \boldsymbol{\theta}\right)$ for the estimation of the parameters $\boldsymbol{\theta}$ is the joint PDF of all samples giving

$$
\ell(\boldsymbol{\theta} \mid \boldsymbol{x})=p(\boldsymbol{x} \mid \boldsymbol{\theta})=\prod_{i=1}^{n} p\left(x_{i} \mid \boldsymbol{\theta}\right) .
$$

Assuming normally distributed and independent input quantities for the dynamic torque application described (i.e. for all of the measurement channels merged in the data vector and therefore for both, input and output quantities of the measured transfer functions); the likelihood function becomes

$$
\ell\left(\boldsymbol{\theta}_{1}, \boldsymbol{\theta}_{2} \mid \boldsymbol{Y}_{\mathrm{D}}(\mathrm{i} \omega)\right) \propto \prod_{i=1}^{n} \mathrm{e}^{-\frac{\left(\boldsymbol{Y}_{\mathrm{D}, i}\left(\mathrm{i} \omega_{i}\right)-\boldsymbol{G}\left(\mathrm{i} \omega_{i}, \boldsymbol{\theta}_{1}, \boldsymbol{\theta}_{2}\right)\right)^{2}}{2 \boldsymbol{u}_{i}^{2}}} .
$$

with the measurement data vector $\boldsymbol{Y}_{\mathrm{D}, i}$ at the corresponding angular frequency $\omega_{i}$ of each data point, the corresponding measurement uncertainty vector $\boldsymbol{u}_{i}$, and the model function $\boldsymbol{G}$. The likelihood function is maximised for the parameter estimation. The estimated parameters $\widehat{\boldsymbol{\theta}}_{2}$ are given by

$$
\widehat{\boldsymbol{\theta}}_{2}=\arg \max _{\widehat{\boldsymbol{\theta}}_{2}} \ell\left(\boldsymbol{\theta}_{1}, \boldsymbol{\theta}_{2} \mid \boldsymbol{Y}_{\mathrm{D}}(\mathrm{i} \omega)\right) \text {. }
$$

The maximum likelihood estimator with a likelihood function as given in (10) can be reduced to a weighted least squares (WLS) estimator [7]. Then, the parameter estimation can be carried out as follows

$\widehat{\boldsymbol{\theta}}_{2}=\arg \min _{\widehat{\boldsymbol{\theta}}_{2}} \sum_{i=1}^{n} \frac{\left(\boldsymbol{Y}_{\mathrm{D}, i}\left(\mathrm{i} \omega_{i}\right)-\boldsymbol{G}\left(\mathrm{i} \omega_{i}, \boldsymbol{\theta}_{1}, \boldsymbol{\theta}_{2}\right)\right)^{2}}{2 \boldsymbol{u}_{i}^{2}}$, applying one of the widely available WLS estimators. For the given application, the induced errors are based on the different measurement quantities and need to be quantified by a measurement uncertainty evaluation. Based on this analysis, a correct weighting of the input measurement channels can be carried out.

The uncertainties of the estimated parameters cannot be calculated directly for the frequentist approaches. The calculation of lower uncertainty limits or of confidence intervals is not applicable for the dynamic torque calibration, because the influences of the uncertainties of the parameters of the measurement $\left(p\left(\boldsymbol{\theta}_{1}\right)\right)$ and influences due to mounting and dismounting of the DUT are invisible for the estimator.

Therefore, the uncertainty evaluation of the estimated parameters will be carried out according to the recommendations of the Guide to the expression of uncertainty in measurement [8] (GUM) and its Supplements 1 [9] and 2 [10], respectively. A Monte Carlo simulation with all input PDFs will be carried out to evaluate the uncertainty of the estimated parameters. The estimation of the uncertainties will not be described in this contribution, but will be part of a dedicated publication.

Differently from the frequentist approach, a parameter estimation based on Bayes' statistics assumes all parameters to be uncertain. Based on Bayes' theorem, the a posteriori PDF follows from the a priori PDF, the evidence's PDF and the likelihood (cf.(9)) giving

$p($ a posteriori $)=\frac{\text { likelihood } \cdot p(\text { a priori })}{p(\text { evidence })}$.

For the parameter estimation of torque transducers, the latter equation leads to

$p\left(\boldsymbol{\theta}_{1}, \widehat{\boldsymbol{\theta}}_{2} \mid \boldsymbol{Y}_{\mathrm{D}}(\mathrm{i} \omega)\right)=\frac{\ell\left(\boldsymbol{\theta}_{1}, \boldsymbol{\theta}_{2} \mid \boldsymbol{Y}_{\mathrm{D}}(\mathrm{i} \omega)\right) p_{0}\left(\boldsymbol{\theta}_{1}\right) p_{0}\left(\boldsymbol{\theta}_{2}\right)}{p\left(\boldsymbol{Y}_{\mathrm{D}}(\mathrm{i} \omega)\right)}$.

It becomes obvious that uncertainties are inherent in a parameter estimation by means of a Bayes' estimator, including uncertainty contributions of the parameters of the measuring device $p_{0}\left(\boldsymbol{\theta}_{1}\right)$, the contributions of the uncertainty of the measurement data, and contributions of prior knowledge of the parameters to be estimated $p_{0}\left(\boldsymbol{\theta}_{2}\right)$. The prior distribution of the parameters to be estimated does not need to be known exactly prior to the estimation (although this is mentioned accordingly in literature [7]); instead reasonable initial PDFs should be available.

Despite all of its advantages, the Bayes' estimator is still rarely used for parameter estimation of mechanical systems. It requires much more effort than LS approaches to be implemented, because it has to be developed individually for each application, whereas for the least-squares-based approaches, an application of the widely available LS algorithms is possible. This effort is the reason why this paper focuses on the least squares approaches in a first step. However, it is planned to develop a Bayes' estimator for the application to dynamic measurements of mechanical quantities in the future.

\section{IMPLEMENTATION OF THE MODEL PARAMETER ESTIMATION}

The parameter estimation based on the measurement data is implemented in Mathworks Matlab and in the open source scientific computing software GNU Octave. The parameter estimation uses a maximum likelihood estimator. This estimator is implemented as a weighted least squares estimator assuming normally distributed and independent input quantities (cf. equation (12)). 
Both transfer functions (see equations (6), (7)) are used jointly for the parameter estimation, i.e. one set of parameters is estimated based on the two measured transfer functions.

Due to the fact that the inverse transfer function equations are more handy than the non-inverted equations (cf. [6]), the implementation of the parameter estimation is based on $H_{\text {top }}^{-1}(\mathrm{i} \omega)$ and $H_{\text {bott }}^{-1}(\mathrm{i} \omega)$.

The weighting of the different data points of each transfer function is based on two conditions:

1. Each transfer function is weighted inversely proportional to its measurement uncertainty. This measurement uncertainty is based on the uncertainty contributions of the incorporated measurement channels. The uncertainty $u$ of each transfer function is calculated by means of a quadratic summation of the two different uncertainty contributions of the input quantities for the transfer function calculation giving $u=\sqrt{u_{1}^{2}+u_{2}^{2}}$. These uncertainty contributions were estimated for magnitude and phase of the transfer functions.

2. The uncertainty of each measurement value is taken into account by evaluating the covariance-variance-matrix as outcome of each sine approximation applied to the time series data. The variances for the approximated parameters (the diagonal elements of the covariance-variance-matrix) are normalised and inverted for the weighting.

The weights $w$ for each data point of each transfer function were calculated by multiplying the two mentioned weighting factors correspondingly for the real $\left(w_{\Re}\right)$ and imaginary part $\left(w_{\Im}\right)$. The equations representing the model parameters within the transfer functions are nonlinear in their parameters. Therefore, only iterative algorithms for nonlinear regression were applicable. To avoid complex numbers in the results for the model parameters, it was necessary to constrain the algorithm to real numbers in the parameter vector. To this end, one set of approximated parameters was calculated by means of the real $(\mathfrak{R})$ and imaginary parts $(\mathfrak{I})$ of the two complex inverse transfer functions giving $\mathfrak{R}\left(H_{\text {top }}^{-1}(\mathrm{i} \omega)\right), \quad \mathfrak{I}\left(H_{\text {top }}^{-1}(\mathrm{i} \omega)\right)$, $\mathfrak{R}\left(H_{\text {bott }}^{-1}(\mathrm{i} \omega)\right), \mathfrak{J}\left(H_{\text {bott }}^{-1}(\mathrm{i} \omega)\right)$.

The resulting model function $\boldsymbol{G}$ follows as

$\boldsymbol{G}\left(\boldsymbol{X}, \boldsymbol{\theta}_{1}, \boldsymbol{\theta}_{2}\right)=\boldsymbol{x}_{1} \cdot \mathfrak{R}\left(H_{\mathrm{top}}^{-1}\left(\boldsymbol{x}_{\omega}, \boldsymbol{\theta}_{1}, \boldsymbol{\theta}_{2}\right)\right)+\boldsymbol{x}_{2}$.

$\mathfrak{I}\left(H_{\text {top }}^{-1}\left(\boldsymbol{x}_{\omega}, \boldsymbol{\theta}_{1}, \boldsymbol{\theta}_{2}\right)\right)+\boldsymbol{x}_{3} \cdot \mathfrak{R}\left(H_{\mathrm{bott}}^{-1}\left(\boldsymbol{x}_{\omega}, \boldsymbol{\theta}_{1}, \boldsymbol{\theta}_{2}\right)\right)+$

$\boldsymbol{x}_{4} \cdot \mathfrak{I}\left(H_{\text {bott }}^{-1}\left(\boldsymbol{x}_{\omega}, \boldsymbol{\theta}_{1}, \boldsymbol{\theta}_{2}\right)\right)$,

with the assignment vectors $\boldsymbol{x}_{1}, \boldsymbol{x}_{2}, \boldsymbol{x}_{3}, \boldsymbol{x}_{4}$ and the frequency vector $\boldsymbol{x}_{\omega}$. All vectors have the length $4 n$ and are summarised in the matrix $\boldsymbol{X}_{\mathrm{A}}$ giving

$X_{\mathrm{A}}=\left[x_{\omega}, x_{1}, x_{2}, x_{3}, x_{4}\right]$,

$\boldsymbol{X}_{\mathrm{A}}=\left[\begin{array}{ccccc}\omega_{11} & 1 & 0 & 0 & 0 \\ \vdots & 1 & 0 & 0 & 0 \\ \omega_{1 n} & 1 & 0 & 0 & 0 \\ \omega_{21} & 0 & 1 & 0 & 0 \\ \vdots & 0 & 1 & 0 & 0 \\ \omega_{2 n} & 0 & 1 & 0 & 0 \\ \omega_{31} & 0 & 0 & 1 & 0 \\ \vdots & 0 & 0 & 1 & 0 \\ \omega_{3 n} & 0 & 0 & 1 & 0 \\ \omega_{41} & 0 & 0 & 0 & 1 \\ \vdots & 0 & 0 & 0 & 1 \\ \omega_{4 n} & 0 & 0 & 0 & 1\end{array}\right]$.

The data vector $\boldsymbol{Y}_{\mathrm{D}}$ follows as
$\boldsymbol{Y}_{\mathrm{D}}\left(\boldsymbol{x}_{\omega}\right)=\left[\begin{array}{c}\mathfrak{R}\left(H_{\mathrm{top}, \mathrm{M}, 1}^{-1}\right) \\ \vdots \\ \mathfrak{R}\left(H_{\mathrm{top}, \mathrm{M}, n}^{-1}\right) \\ \mathfrak{J}\left(H_{\mathrm{top}, \mathrm{M}, 1}^{-1}\right) \\ \vdots \\ \mathfrak{J}\left(H_{\mathrm{top}, \mathrm{M}, n}^{-1}\right) \\ \mathfrak{R}\left(H_{\mathrm{bott}, \mathrm{M}, 1}^{-1}\right) \\ \vdots \\ \mathfrak{R}\left(H_{\mathrm{bott}, \mathrm{M}, n}^{-1}\right) \\ \mathfrak{J}\left(H_{\mathrm{bott}, \mathrm{M}, 1}^{-1}\right) \\ \vdots \\ \mathfrak{J}\left(H_{\mathrm{bott}, \mathrm{M}, n}^{-1}\right)\end{array}\right]$.

The weights for each data point are summarised in the weighting vector $\boldsymbol{w}$ accordingly, giving

$\boldsymbol{W}=\left[\begin{array}{c}w_{\mathfrak{R}\left(H_{\mathrm{top}, 1}^{-1}\right)} \\ \vdots \\ w_{\mathfrak{R}\left(H_{\mathrm{top}, n}^{-1}\right)} \\ w_{\Im\left(H_{\mathrm{top}, 1}^{-1}\right)} \\ \vdots \\ w_{\Im\left(H_{\mathrm{top}, n}^{-1}\right)} \\ w_{\mathfrak{R}\left(H_{\mathrm{bott}, 1}^{-1}\right)} \\ \vdots \\ w_{\mathfrak{R}\left(H_{\mathrm{bott}, n}^{-1}\right)} \\ w_{\Im\left(H_{\mathrm{bott}, 1}^{-1}\right)} \\ \vdots \\ w_{\Im\left(H_{\mathrm{bott}, n}^{-1}\right)}\end{array}\right]$.

The cost function

$\boldsymbol{K}\left(\boldsymbol{x}_{\omega}, \boldsymbol{\theta}_{1}, \boldsymbol{\theta}_{2}\right)=\left(\boldsymbol{Y}_{\mathrm{D}}\left(\boldsymbol{x}_{\omega}\right)-\boldsymbol{G}\left(\boldsymbol{X}_{\mathrm{A}}, \boldsymbol{\theta}_{1}, \boldsymbol{\theta}_{2}\right)\right) \cdot \boldsymbol{w}$

corresponds directly with (12) and is minimised for the parameter estimation.

\section{MEASUREMENT RESULTS}

The analysis of the feasibility of the parameter estimation was carried out with one HBM T5 $10 \mathrm{~N} \cdot \mathrm{m}$ shaft type torque transducer (depicted in Figure 5). The chosen transducer is a passive strain gauge transducer, the bridge signals are transmitted by means of slip rings. The signal conditioning electronics, data acquisition systems, and filters have been calibrated dynamically prior to the measurements. The electrical influences of these components of the measurement chain have been compensated for [11].

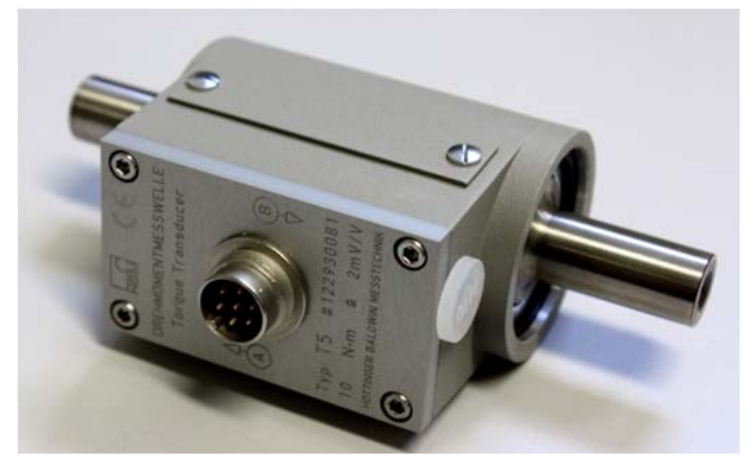

Figure 5. Torque transducer HBM T5 - nominal torque $10 \mathrm{~N} \cdot \mathrm{m}$. 
For the measurement, sinusoidal excitations in a frequency range of about $10 \mathrm{~Hz}$ to $1 \mathrm{kHz}$ can be applied. It is advantageous for the model parameter identification to include frequencies which are as high as possible, because the sensitivity to parameter changes increases with higher frequencies [6]. However, the upper frequency limit for the measurement is not only limited by the capabilities of the rotational exciter, but by the dynamic behaviour of the whole shaft assembly.

Beyond the (first) resonance frequency of the shaft assembly, the components arranged above the dominant spring element will be dynamically decoupled. The higher the excitation frequency is with respect to this resonance frequency, and the lower the damping of the system, the stronger the decoupling is.

In practice, the angular acceleration at the top of the measuring device ( $\ddot{\varphi}_{\mathrm{M}}$, cf. Figure 1) will become too small and too disturbed to be reasonably measured for frequencies far beyond the resonance frequency. For the investigated transducer HBM T5 $10 \mathrm{~N} \cdot \mathrm{m}$, the upper frequency limit for the measurements was reached somewhat above $300 \mathrm{~Hz}$.

The dynamic torque magnitude generated by the dynamic torque calibration device is relatively small at present and is in the range of about $0.5 \mathrm{~N} \cdot \mathrm{m}$ to $1 \mathrm{~N} \cdot \mathrm{m}$.

Figure 6 shows the magnitude and phase responses of the complex transfer functions of the top and of the bottom of the measuring device $H_{\text {top }}(\mathrm{i} \omega)$ and $H_{\text {bott }}(\mathrm{i} \omega)$ for both measurement data and estimation.

The measurement results show that the deviations from the estimated values of the top transfer function increase for frequencies higher than the resonance frequency of the system. This is caused by the mentioned increasing decoupling of the top part of the shaft assembly for frequencies beyond the resonance frequency.

The results of the model parameter estimation show a fairly good agreement of the measurement data and the outcome of the regression, which proves the validity of the model assumptions for the transducer and for the measuring device. The real and imaginary parts of the two inverse transfer functions are given in Figure 7. These are the transfer functions, with which the parameter estimation was carried out.

The real parts of the two transfer functions show very good agreement of measured values and fit, however, the imaginary
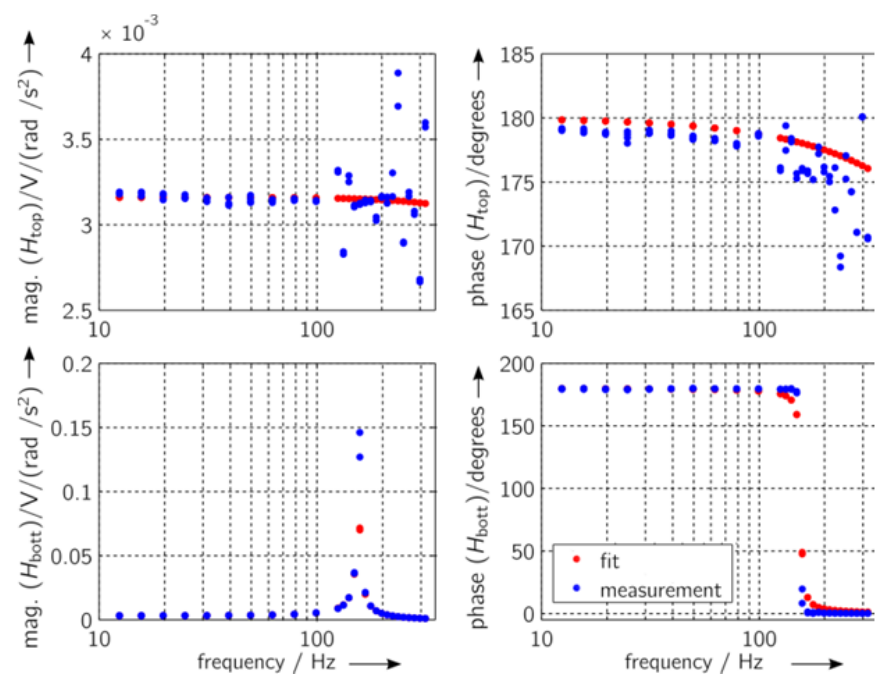

Figure 6. Measurement data of the HBM T5 transducer (blue) and fit result (red) for $H_{\text {top }}(\mathrm{i} \omega)$ (top) and $H_{\text {bott }}(\mathrm{i} \omega)$ (bottom) in magnitude and phase.
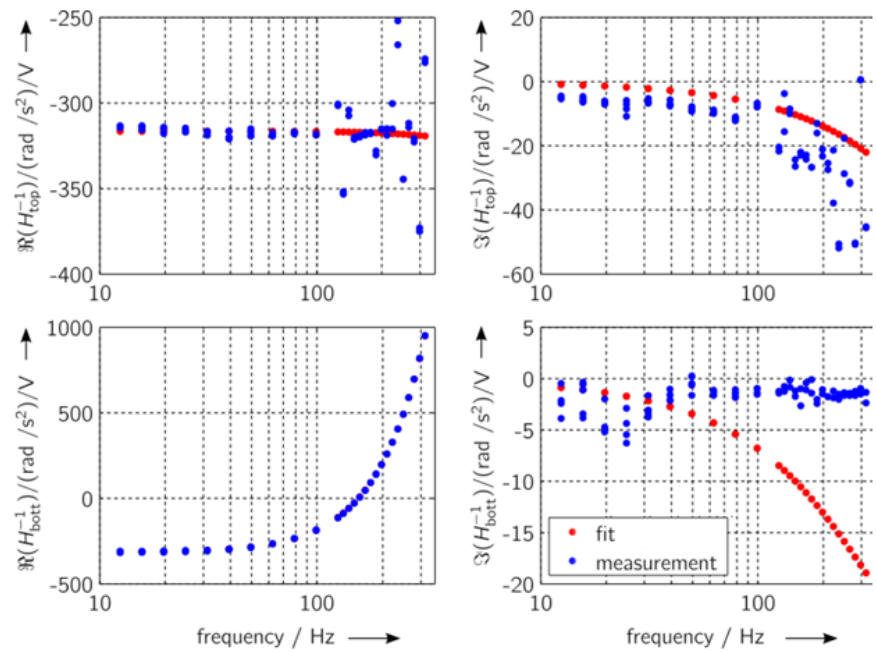

Figure 7. Measurement data of the HBM T5 transducer (blue) and fit result

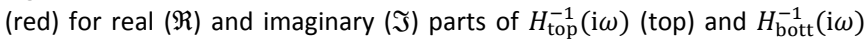
(bottom).

parts reveal discrepancies between observed values and the regression data.

As a next step, the correctness of the identified parameters was assessed. Agreement between measurement and parameter estimation results does not automatically yield parameters that correspond with the transducer's real properties. This may happen if the model assumption and the mechanical system do not agree, but somehow a fit is possible, e.g. if some of the parameters of the measuring device are not correct or the transducer's mechanical design is not sufficiently represented by the model.

Therefore, a validation of the parameter estimation results was carried out by means of independent measurements and additional available information. This validation - as described in the next paragraphs - is an intermediate step towards a validation incorporating measurement uncertainties of all available sources. The agreement of measurement data and the results of the parameter identification will be analysed in terms of the uncertainties of both measurement data and model parameters in a subsequent publication. Due to the fact that the parameters of the measuring device - which were determined with assigned measurement uncertainties - are also incorporated in the transfer function equations, a comparison of identified parameters and measurement data considering only the measurement uncertainties of the measurement data is not sufficient.

\section{VALIDATION BY MEANS OF INDEPENDENT MEASUREMENTS}

The identified model parameters of the investigated transducer HBM T5 are given in Table 2. The parameters identified describe the mechanical properties of the transducer, which can be found - to some extent - in technical data sheets. However, this information is not reliable, because neither can one ascertain how these specifications are derived, nor are any uncertainties for the given specifications available. Nevertheless, the data sheet information of the HBM T5 transducer [12] was compared with all other results.

Additionally, some parameters of the investigated transducer were determined by independent static measurements. These measurements were carried out with the auxiliary measurement 
Table 2. Results of the parameter identification.

\begin{tabular}{l|cccc} 
& $J_{\mathrm{H}}$ & $c_{\mathrm{T}}$ & $d_{\mathrm{T}}$ & $J_{\mathrm{B}}$ \\
\hline measurement 1 & $4 \cdot 10^{-11} \mathrm{~kg} \cdot \mathrm{mm}^{2}$ & $603 \mathrm{~N} \cdot \mathrm{m} / \mathrm{rad}$ & $3 \cdot 10^{-2} \mathrm{~N} \cdot \mathrm{m} \cdot \mathrm{s} / \mathrm{rad}$ & $446 \mathrm{~kg} \cdot \mathrm{mm}{ }^{2}$ \\
measurement 2 & $8 \cdot 10^{-11} \mathrm{~kg} \cdot \mathrm{mm}^{2}$ & $607 \mathrm{~N} \cdot \mathrm{m} / \mathrm{rad}$ & $1 \cdot 10^{-2} \mathrm{~N} \cdot \mathrm{m} \cdot \mathrm{s} / \mathrm{rad}$ & $272 \mathrm{~kg} \cdot \mathrm{mm}{ }^{2}$
\end{tabular}

set-ups already used for the determination of the mechanical properties of the measuring device [3]. While the torsional stiffness was measured without any modifications of the transducer, the friction of the slip rings and of the ball bearings made a measurement of the mass moment of inertia of the transducer's rotor by means of a pendulum set-up infeasible. Instead, a bare rotor shaft without bearings of a similar transducer (same type and same torque capacity) was used for that purpose.

It was not possible to validate the damping coefficient by independent measurements, but due to the known low damping of transducers and the large resonance rise experienced (cf. Figure 6), the influence of the damping can be assumed to be low and therefore to be less important for the dynamic behaviour of the transducer.

The results of the measurements and of the manufacturer's specifications are given in Table 3.

Table 2 and Table 3 show that the data sheet specifications of the torsional stiffness agree very well with the experimental results from the auxiliary measurement, but the results from the mass moment of inertia deviate significantly.

Two issues exist for the validation of the results of the mass moment of inertia parameters of the transducer:

1. Only the overall mass moment of inertia $J=J_{\mathrm{H}}+J_{\mathrm{B}}$ can be determined by independent measurements. The top mass moment of inertia $J_{\mathrm{H}}$ was roughly estimated by means of the geometry of the rotor and with an assumed generic density of steel of $\rho \approx 7.8 \mathrm{~g} / \mathrm{cm}^{3}$ giving $J_{\mathrm{H}} \sim 6 \mathrm{~kg} \cdot \mathrm{mm}^{2}$. This number is so small that even with the set-up dedicated for the determination of mass moment of inertia, the resulting measurement uncertainty would be in the same magnitude as the measured quantity.

2. The identified mass moment of inertia parameter of the bottom part of the transducer $\left(J_{\mathrm{B}}\right)$ is significantly larger than expected. This deviation is most likely caused by the friction of the slip rings, which are arranged below the sensing element of the transducer.

\section{VALIDATION BY MEANS OF A VARIATION OF THE PROPERTIES OF THE DEVICE UNDER TEST}

To overcome these issues, an alternative validation approach was applied. The existing drive shaft assembly was modified with two different additional mass moment of inertia elements, which could be added to $J_{\mathrm{H}}$. The design of these mass bodies was chosen in such a way that a rigid connection minimises

Table 3. Manufacturer's specifications and independent measurement results for mass moment of inertia and torsional stiffness with assigned expanded relative measurement uncertainties $U_{\text {rel }}$.

\begin{tabular}{l|ll} 
& $J=J_{\mathrm{H}}+J_{\mathrm{B}}$ & $c=c_{\mathrm{T}}$ \\
\hline $\begin{array}{l}\text { specifications } \\
\text { from data sheet }\end{array}$ & $41 \mathrm{~kg} \cdot \mathrm{mm}^{2}$ & $640 \mathrm{~N} \cdot \mathrm{m} / \mathrm{rad}$ \\
{$[12]$} & & \\
measurement & $38.6 \mathrm{~kg} \cdot \mathrm{mm}^{2}$ & $638.29 \mathrm{~N} \cdot \mathrm{m} / \mathrm{rad}$ \\
results & $U_{\mathrm{rel}}(k=2)=9.0 \%$ & $U_{\mathrm{rel}}(k=2)=0.1 \%$
\end{tabular}

influences due to the mounting of the adapter. The mass moment of inertia of the mass bodies was measured independently in the same way as for the rotor of the torque transducer.

One mass body is based on a modified coupling element (depicted in Figure 8); it has a mass moment of inertia of $J=878.86 \mathrm{~kg} \cdot \mathrm{mm}^{2}\left(U_{\mathrm{rel}}(k=2)=5 \cdot 10^{-4}\right)$. Furthermore one mass body is an aluminium ring (depicted in Figure 9) with a mass moment of inertia of $=295.64 \mathrm{~kg} \cdot \mathrm{mm}^{2} \quad\left(U_{\text {rel }}(k=2)\right.$ $=1 \cdot 10^{-3}$ ).

Both mass bodies were used for validation measurements simulating a transducer with a correspondingly larger $J_{\mathrm{H}}$. The added mass moment of inertia in conjunction with the unchanged torsional stiffness of the torque transducer under test leads to lower resonance frequencies, and therefore to a decreased upper frequency limit for the dynamic measurements.

The measurement results for the mass moment of inertia element using the coupling element $\left(J \approx 900 \mathrm{~kg} \cdot \mathrm{mm}^{2}\right)$ are depicted in Figure 10, and the results for the aluminium ring $\left(J \approx 300 \mathrm{~kg} \cdot \mathrm{mm}^{2}\right)$ are shown in Figure 11 . The resulting magnitude and phase responses of the parameters identified agree well with the corresponding measurement result. The parameters identified are given in Table 4.

For both measurements with additional mass bodies, the parameters identified agree very well with the expectations. The mass moment of inertia of the top $J_{\mathrm{H}}$ agrees excellently with the

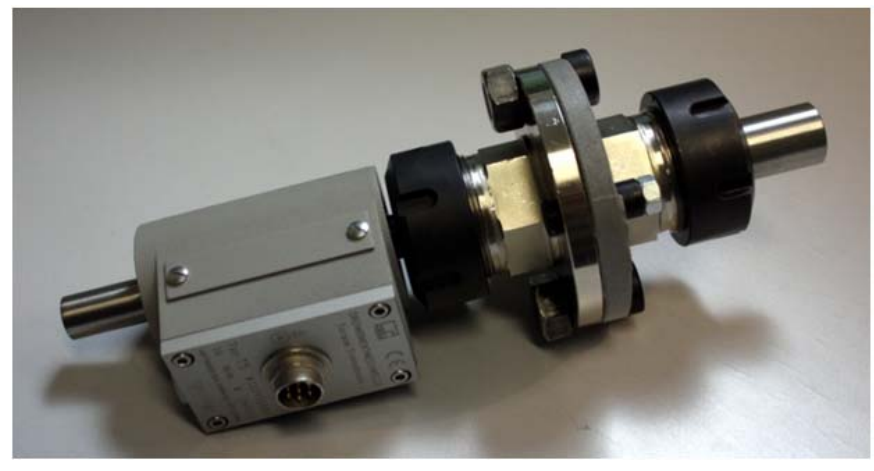

Figure 8. Torque transducer HBM T5 (left) with the mass moment of inertia element $J \approx 900 \mathrm{~kg} \cdot \mathrm{mm}^{2}$ (right).

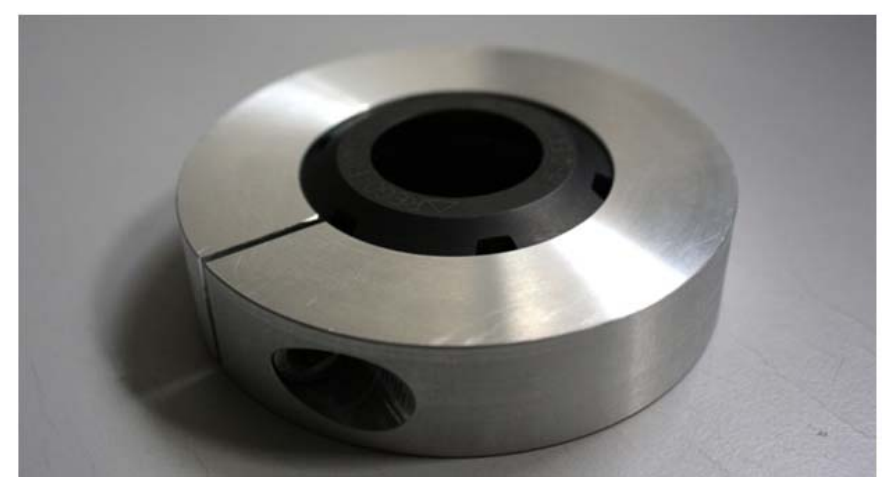

Figure 9. Moment of inertia element $J \approx 300 \mathrm{~kg} \cdot \mathrm{mm}^{2}$ mounted on a clamping nut. 
Table 4. Results of the parameter identification with additional mass moment of inertia elements.

\begin{tabular}{l|cccc} 
& $J_{\mathrm{H}}$ & $c_{\mathrm{T}}$ & $d_{\mathrm{T}}$ \\
\hline $\mathrm{HBM}$ T5 with mass body & $945 \mathrm{~kg} \cdot \mathrm{mm}^{2}$ & $637 \mathrm{~N} \cdot \mathrm{m} / \mathrm{rad}$ & $2 \cdot 10^{-14} \mathrm{~N} \cdot \mathrm{m} \cdot \mathrm{s} / \mathrm{rad}$ & $874 \mathrm{~kg} \cdot \mathrm{mm}{ }^{2}$ \\
$J \approx 900 \mathrm{~kg} \cdot \mathrm{mm}^{2}$ & & & & \\
$\mathrm{HBM} \mathrm{T} 5$ with mass body & $311 \mathrm{~kg} \cdot \mathrm{mm}^{2}$ & $635 \mathrm{~N} \cdot \mathrm{m} / \mathrm{rad}$ & $9 \cdot 10^{-3} \mathrm{~N} \cdot \mathrm{m} \cdot \mathrm{s} / \mathrm{rad}$ & $239 \mathrm{~kg} \cdot \mathrm{mm}{ }^{2}$
\end{tabular}
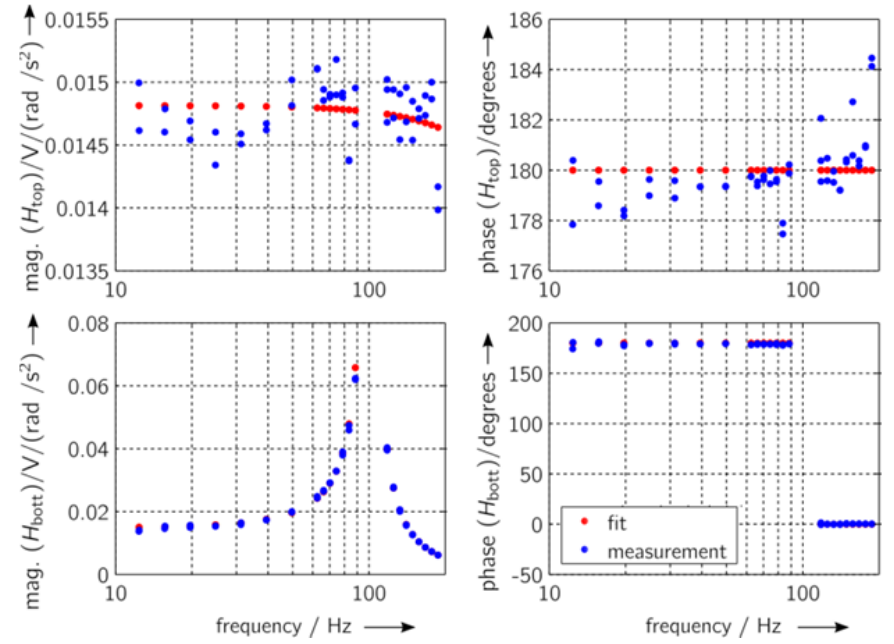

Figure 10. Measurement data of the HBM T5 transducer with additional mass moment of inertia element $\left(J \approx 900 \mathrm{~kg} \cdot \mathrm{mm}^{2}\right.$ ) (blue) and fit result (red) for $H_{\text {top }}(\mathrm{i} \omega)$ (top) and $H_{\text {bott }}(\mathrm{i} \omega)$ (bottom) in magnitude and phase.
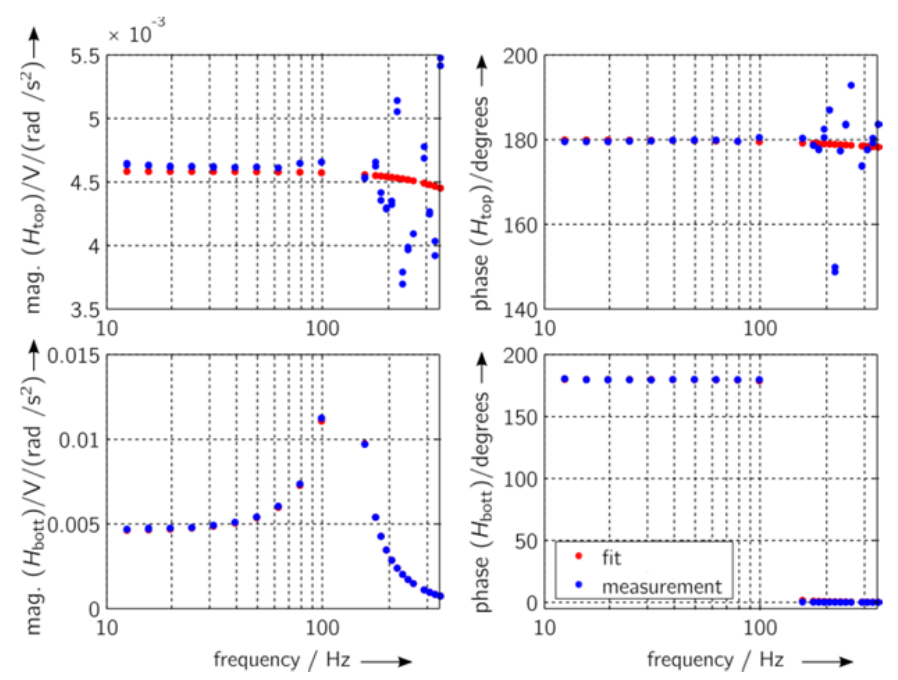

Figure 11. Measurement data of HBM T5 with additional mass moment of inertia element $\left(J \approx 300 \mathrm{~kg} \cdot \mathrm{mm}^{2}\right.$ ) (blue) and fit result (red) for $H_{\text {top }}(\mathrm{i} \omega$ ) (top) and $H_{\text {bott }}(\mathrm{i} \omega)$ (bottom) in magnitude and phase.

added mass moments of inertia. The torsional stiffness parameter $c_{\mathrm{T}}$ matches the independent measurements even better than with the measurements of the transducer alone. $J_{\mathrm{B}}$ is in a range similar to that of the measurements without additional mass bodies due to the influences from the friction of the slip rings. However, the reduced excitation frequency range causes higher uncertainties of the identified parameters, which will be described in a later publication.

\section{SUMMARY AND OUTLOOK}

A model-based approach enables the identification of the dynamic properties of torque transducers from measurement data. The model is linear and time-invariant and consists of known model properties of the measuring device and unknown model properties of the transducer under test. The parameter identification is carried out using the acquired measurement data which was corrected for influences from signal conditioning electronics and the data acquisition system. The parameters are estimated by means of a maximum likelihood estimator.

Three signals are acquired during the calibration measurements. Based on this data, two complex transfer functions are calculated. A nonlinear regression is carried out based on the two transfer functions to estimate a set of common parameters. These parameters describe the dynamic behaviour of the torque transducers under test.

The measurement results of a first transducer show good agreement to the model assumptions. Remaining discrepancies in the imaginary parts of the two transfer functions require further investigation.

The parameter estimation based on the measurement data gives reasonable results. A validation of the identified parameters was carried out by means of independent measurements, as well as with a modified measurement set-up. The validation results agree very well with the identified parameters of the transducer and therefore confirm the results of the parameter estimation.

The described validation is an intermediate step towards model-based dynamic torque calibration. The estimation of the uncertainties of the measurement and of the parameter identification, as well as the comparison of these results with independent measurements, is beyond the scope of this paper and will be covered by a dedicated publication in future.

\section{ACKNOWLEGDEMENT}

The author would like to thank the company HBM - in particular Dr André Schäfer - for the items provided on loan (sometimes at short notice), which reduced the time needed for the validation of the parameter estimation results.

\section{REFERENCES}

[1] L. Klaus, Th. Bruns and M. Kobusch, "Dynamic Torque Calibration - Necessity and Outline of a Model-Based Approach", Proc. of 5th International Competition Best Young Metrologist of COOMET 2013, pp. 61-64, Braunschweig, Germany, June 2013, DOI: $10.7795 / 810.20140929$.

[2] C. Bartoli et al., "Traceable Dynamic Measurement of Mechanical Quantities: Objectives and First Results of this European Project", International Journal of Metrology and Quality Engineering, vol. 3 (3), pp. 127-137, May 2013, DOI: 10.1051/ijmqe/2012020.

[3] L. Klaus, "Identification of Model Parameters of a Partially Unknown Linear Mechanical System from Measurement Data", Proc. of XXI IMEKO World Congress, Prague, Czech Republic, Sep. 2015, http://www.imeko.org/publications/wc-2015/ IMEKO-WC-2015-TC3-050.pdf.

[4] L. Klaus, Th. Bruns and M. Kobusch, "Modelling of a dynamic torque calibration device and determination of model 
parameters", Acta IMEKO, vol. 3 (2), pp. 14-18, June 2014 DOI: $10.21014 /$ acta imeko.v3i2.79.

[5] L. Klaus and M. Kobusch, "Experimental Method for the NonContact Measurement of Rotational Damping", Proc. of Joint IMEKO International TC3, TC5 and TC22 Conference 2014, Cape Town, South Africa, February 2014, http://www.imeko.org/publications/tc22-2014/IMEKO-TC3TC22-2014-003.pdf.

[6] L. Klaus, B. Arendacká, M. Kobusch and Th. Bruns, "Dynamic Torque Calibration by Means of Model Parameter Identification", Acta IMEKO, vol. 4 (2), pp. 39-44, June 2015, DOI: $10.21014 /$ acta imeko.v4i2.211.

[7] J. Schoukens, R. Pintelon, Identification of Linear Systems - A Practical Guideline, Pergamon Press, Oxford, 1991.

[8] Bureau International des Poids et Mesures, Evaluation of measurement data - Guide to the expression of uncertainty in measurement $=$ Évaluation des données de mesure - Guide pour l'expression de l'incertitude de mesure, 2008.

[9] Bureau International des Poids et Mesures, Evaluation of measurement data - Supplement 1 to the "Guide to the expression of uncertainty in measurement" - Propagation of distributions using a Monte Carlo method = Évaluation des données de mesure - Supplément 1 du "Guide pour l'expression de l'incertitude de mesure" - Propagation de distributions par une méthode de Monte Carlo, 2008.

[10] Bureau International des Poids et Mesures, Evaluation of measurement data - Supplement 2 to the "Guide to the expression of uncertainty in measurement" - Extension to any number of output quantities = Évaluation des données de mesure - Supplément 2 du "Guide pour l'expression de l'incertitude de mesure" - Extension à un nombre quelconque degrandeurs de sortie, 2011.

[11] L. Klaus, Th. Bruns and H. Volkers, "Calibration of bridge-, charge- and voltage amplifiers for dynamic measurement applications", Metrologia, vol. 52(1), pp. 72-81, February 2015, DOI: $10.1088 / 0026-1394 / 52 / 1 / 72$.

[12] Hottinger Baldwin Messtechnik GmbH, "T5 Torque Transducer Data Sheet", Version B0071-1.2, 2004. 